\title{
Single Mode Lasing in InGaAsP/InP Semiconductor Coupled Square Ring Cavities
}

\author{
Kyung-Sook Hyun*, Taekyu Lee, and Hee-Jong Moon \\ Department of Optical Engineering, Sejong University, Seoul 143-747, Korea
}

(Received April 5, 2012 : revised April 23, 2012 : accepted May 9, 2012)

\begin{abstract}
This work reports the stability of the resonant characteristics in multimode interferometer coupled square ring semiconductor cavities. Based on the analysis of single square ring cavities, the single mode operations in the multimode interferometer coupled ring cavities are analyzed and the devices are demonstrated on the semiconductor multiple quantum well epitaxial structure. By varying the lasing conditions such as substrate temperature and input pump power, single resonant mode operations are also observed.

Keywords: Square ring cavity, Multi-mode interference, Semiconductor, Single-mode lasing

OCIS codes : (130.3120) Integrated optics devices; (130.5990) Semiconductors; (140.3948) Microcavity devices; (140.3570) Lasers, single-mode
\end{abstract}

\section{INTRODUCTION}

Semiconductor ring cavities offer a large number of applications due to the compact geometry, no need of cleaving and the possibility of integration between active and passive devices [1-10]. Recently, devices with ring cavities have been widely studied and utilized in several different technologies at all levels of cost in fabrication [11-19]. In most semiconductor cavities, the radius should be many times the operating wavelength to attain high quality (Q) factors $[14,21,22]$. In the case of using these cavities, in other words the multiple resonance case, the closely spaced wavelength resonances are inevitably obtained. However, the narrow free spectral range (FSR) of the output spectra is not desirable in most photonic device applications, such as passive filters and laser cavities. Thus, achieving a moderate FSR or a single mode operation in an optical cavity is essential if it is to be used as an integrated optical component.

One of the possible methods to obtain a large FSR is using the coupled cavities to lase only the common resonance mode between two cavities. Usually, the coupling between cavities or with the waveguide needs a very small gap distance down to sub micrometers due to the high lateral confinement by deep etching. Eventually, we would not be able to avoid an intricate process such as e-beam lithography when use the semiconductor materials. This difficulty limits the fabrication and application of semiconductor ring or disk cavities as the integrated device elements. In our previous work [23], single mode lasing was reported in the multimode interferometer (MMI) coupled square ring cavity, which does not use evanescent coupling. In this work, we focused on the stabilities of the single mode behavior in coupled cavities by varying the ambient temperature and input pump powers.

\section{DESIGN, FABRICATION AND MEASUREMENTS}

Before the study of the coupled square ring cavity, the single square ring cavity was fabricated and characterized. In figure 1 (a), the schematic diagram of a single square ring cavity is presented and its waveguide width (w) and cavity length (d) are defined. In addition, the output spectrum of the fabricated single cavity and its microphotograph are shown in figure 1 (b). We are going to briefly describe the design, fabrication and measurement processes of the square ring cavity. Details of the entire processes have been described elsewhere [23].

For the proposed semiconductor cavity fabrication, InP/ InGaAsP based semiconductor epitaxial layers were chosen. The active layers consisted of 6 layers of undoped InGaAsP/ InGaAs multiple quantum well (MQW)s with a center wavelength, $\lambda_{\mathrm{g}}=1.55 \mu \mathrm{m}$ and are sandwiched by $\mathrm{n}$ - and p-type InGaAsP confinement layers. p-InP on active layers

\footnotetext{
*Corresponding author: kshyun@sejong.ac.kr

Color versions of one or more of the figures in this paper are available online.
} 
and $\mathrm{n}$-InP buffer on $\mathrm{InP}$ substrate were used as cladding layers.

The devices are fabricated by a standard III-V compound semiconductor process. Particularly, a careful etching process using $\mathrm{CH}_{4} / \mathrm{H}_{2}$ reactive ion etching is needed to obtain the refined vertical surface of a device. In addition, the etch depths exceeding $4.2 \mu \mathrm{m}$ are controlled to laterally confine the travelling wave and to prevent radiation losses into the substrate.

For a measurement of the cavity properties, an acoustooptic Q-switched $(10 \mathrm{kHz}) \mathrm{Nd}: \mathrm{YVO} 4$ laser, wavelength of $1.06 \mu \mathrm{m}$, is adapted to optically pump MQWs. With this wavelength, some of the pump light penetrated the clad layer and was strongly absorbed by the MQW layers. The lasing signal from the coupled-cavity was collected through a tapered fiber launched around the cavity corner and inclined to cavity plane in order to measure maximum intensity and its spectrum was analyzed by an optical spectrum analyzer (HP 81818).

The waveguide width $(\mathrm{w})$ of the square cavity, illustrated in the inset in figure 1 (b), is $10 \mu \mathrm{m}$ and length of the cavity side (d) is $50 \mu \mathrm{m}$. The square ring cavity has $45^{\circ}$ -

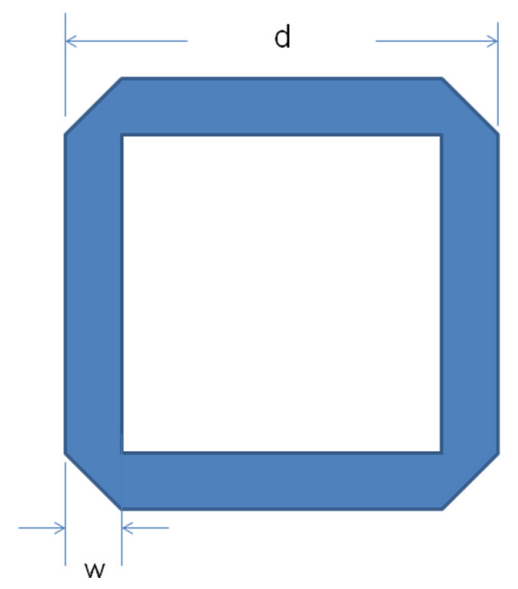

(a)

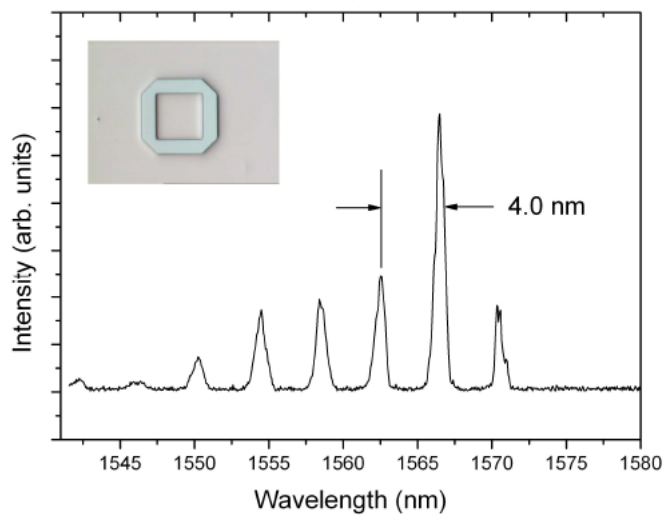

(b)

FIG. 1. (a) Schematic diagram of a square ring cavity. (b) Typical lasing spectrum of the single square ring cavity and its microphotograph in the inset. tilted flat corners. When the corners are symmetrically $45^{\circ}$ -tilted, closed orbits, formed by total reflections with an incident angle $45^{\circ}$ at the corner, are possible. Based on this closed orbit cavity mode, the lasing spectrum of the single square cavity showed multimode and many closely spaced resonance peaks of $4 \mathrm{~nm}$ as shown in figure 1 (b). To support this value, the parameter $\mathrm{m}^{\prime}$ was estimated to be 3.55 at $1550 \mathrm{~nm}$ by the calculation represented as below. The spacing between the multi-resonant wavelengths can be controlled by varying the cavity length. For the calculations of the resonant wavelengths, the optical path length $\mathrm{L}$ can be given as follows;

$$
L=4 m(d-w)
$$

where $m$ is the effective refractive index of the semiconductor materials. In addition, the resonance condition is given by;

$$
L=m\left(\lambda_{n}\right) l=n \lambda
$$

where $n$ is an integer and $l$ is the round trip length. Based on the above equation (1) and equation (2), the adjacent resonant mode wavelength spacing $\left(\Delta \lambda=\lambda_{n-1}-\lambda_{n}\right)$ can be derived as follows;

$$
\Delta \lambda=\frac{\lambda^{2}}{m l\left(1-\frac{d m}{d \lambda} \frac{\lambda}{m}\right)}=\frac{\lambda^{2}}{m^{\prime} l}, \quad \text { where } \quad m^{\prime}=m\left(1-\frac{d m}{d \lambda} \frac{\lambda}{m}\right) \text {. }
$$

This Fabry-Perot-like resonant equation is valid when the cavity sizes are much larger than the operating wavelengths.

Figure 2 exhibits the schematic diagram of the MMI coupled square ring structure. An MMI is proposed to couple the square ring cavity. Unlike the circular cavity, the straight MMI is the best choice to couple two square

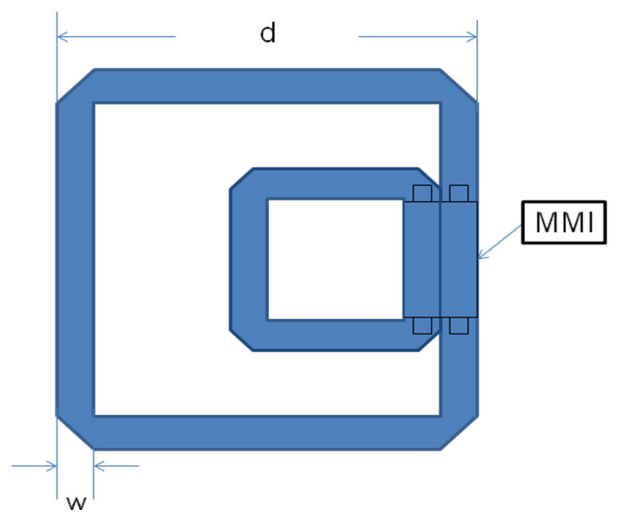

FIG. 2. Schematic diagram of an MMI coupled square ring cavity and the definitions of cavity length (d) and waveguide width $(w)$ is indicated. 
cavities. MMI is known to have self-imaging or power splitting functions by controlling the MMI length [24, 25]. As shown in figure 2, a square cavity rests within a larger square cavity. One side of the smaller cavity lies along a side of the larger cavity. Thus, the coupled waveguide width is twice the square waveguide width and can be regarded as an MMI. When the light enters a coupled waveguide region, it suffers abrupt waveguide broadening. Finally, the light intensities are divided and coupled separately into outside and inside cavity.

The main idea is to use the Vernier effect with which it is possible to enhance only one mode by suppressing every other mode existing in a single square ring cavity. The selected modes that fulfill both cavity resonance conditions are significantly enhanced. As a result, the single mode lasing can be expected through the common resonance of two semiconductor cavities.

\section{RESULTS AND DISCUSSION}

Figure 3 shows the typical lasing spectrum of the fabricated MMI-coupled square cavity by pulsed optical pumping and its logarithmic value of the lased optical intensities in the inset. The device has a specification of an outside cavity length, an inside cavity length and a waveguide width of $50 \mu \mathrm{m}, 20 \mu \mathrm{m}$ and $4 \mu \mathrm{m}$, respectively, and it will be referred to as 50-20-4. From the beam propagation calculation, the coupling ratio into the adjacent cavities was calculated to 0.24 at the center wavelength. Depending on the equations presented above, the FSR of each cavity was calculated as $3.28 \mathrm{~nm}$ for the $50 \mu \mathrm{m}$-cavity and $8.20 \mathrm{~nm}$ for the $20 \mu \mathrm{m}$-cavity.

The illustrated lasing spectrum of the MMI coupled cavity in figure 3 represents single mode operation with full width at half maximum (FWHM) of $0.83 \mathrm{~nm}$ and a side mode suppression ratio (SMSR) of $24.90 \mathrm{~dB}$, which is the

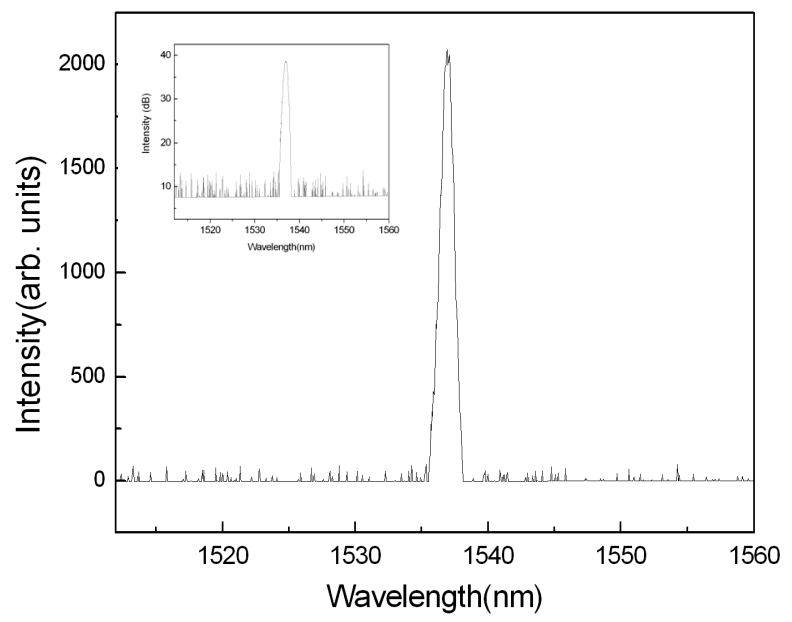

FIG. 3. Single mode lasing spectra of the MMI coupled square ring cavity and its logarithmic values in the inset. best result we measured. The center wavelength was measured as $1537.0 \mathrm{~nm}$, which is in the range of the photoluminescence spectrum of the MQW. From these experimental results, single mode operations can be simply obtained in the MMI coupled square cavity.

Beside the resonant characteristics, it is important to get the information of the stability of the coupled cavity by changing the temperature and pump optical power. In figure 4 , the temperature dependent lasing center wavelengths have been measured for devices with 50-20-6, 50-30-6, and 80-30-6 devices. The temperature of the semiconductor device substrate was controlled by thermoelectric cooler between $20^{\circ} \mathrm{C}$ to $65^{\circ} \mathrm{C}$. Increasing the temperature, the single mode lasing was maintained within the measured temperature range in spite of the red shift of the center wavelength. Mode hopping was also observed but the hopping temperature and the total amount of hopping distance were not fixed. This can be explained by an effective refractive index change in semiconductors according to temperature change. Except for the mode hopping region, the temperature dependent red shift of the wavelength is too small to be considered as a material dispersion. The value of $\sim 1 \mathrm{~nm} /{ }^{\circ} \mathrm{C}$ is comparable to the distributed feedback laser diode [26], which implies that the lasing wavelength is dominantly determined by its structure, not by the material dispersion.

The differences in hopping values categorized by various cavity combinations are summarized in Table 1 . In the order of 80-30-6, 50-30-6, and 50-20-6 devices, the hopping

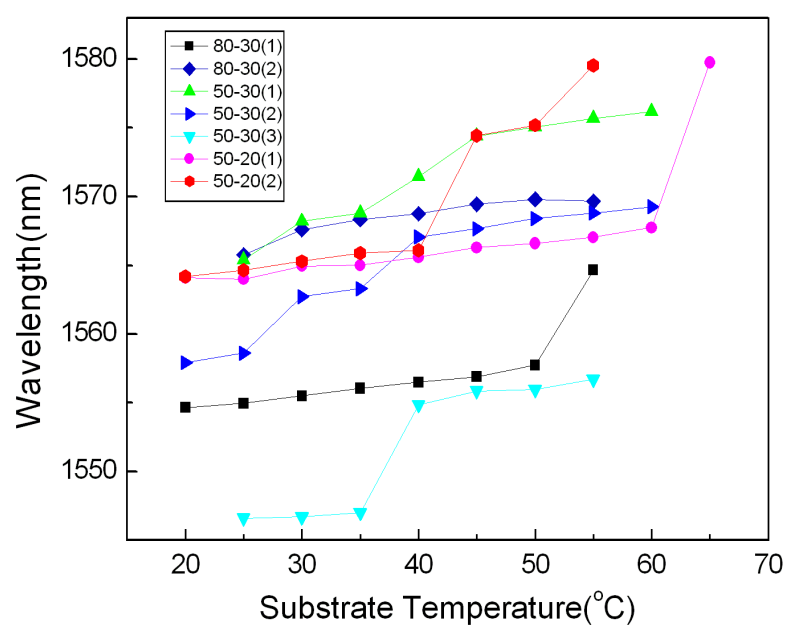

FIG. 4. The center wavelength shifts by varying the substrate temperature for coupled ring cavities. The waveguide width is fixed to $6 \mathrm{~mm}$ while the combinations of the cavity length are varied as 50-20, 50-30 and 80-30. The indices (1), (2) or (3) mean the different devices with the same device structures.

TABLE 1. The average mode hopping distance in wavelength for the various devices are summarized

\begin{tabular}{c|c|c|c}
\hline \hline Coupled Cavity Type & $50-20-6$ & $50-30-6$ & $80-30-6$ \\
\hline Mode Hopping Distance (nm) & 12.3 & 7.8 & 4.4 \\
\hline
\end{tabular}




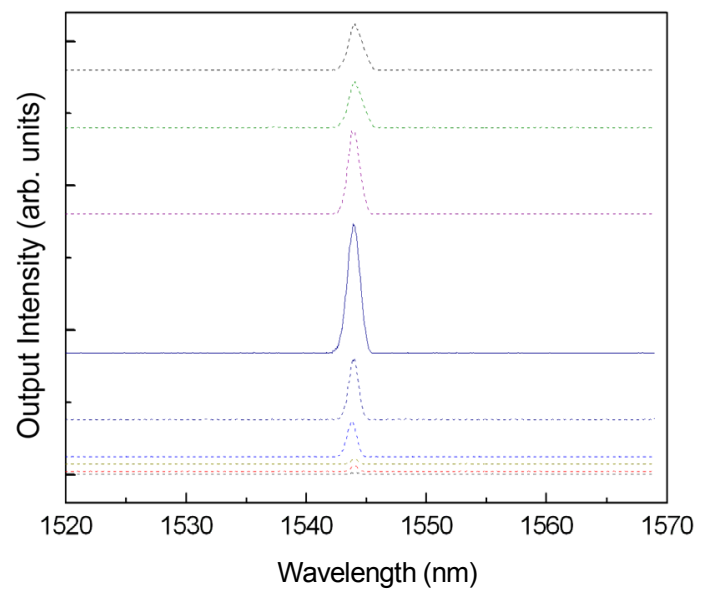

FIG. 5. The lasing spectra of the MMI coupled square ring cavity by increasing the optical pump power.

distance has clearly increased. This can be reasonably understood knowing that the mode spacing of the long cavity is smaller than that of the short cavity. As a result, the hopping of the resonant wavelength can get larger as the device size decreases.

We also investigated the single mode behavior by varying the optical input powers as shown in figure 5. The vertical axis shows linear intensity of the emitted spectra from the cavity. In order to easily compare the lasing characteristics according to pump power, the lasing curves were placed vertically as the pump beam intensity increased.

The input power level was increased several times larger than the threshold average input power level for each measurement step. The threshold pump power of the coupled cavity was measured to be $\sim 2 \mathrm{~mW}$. By increasing the pump power up to $\sim 30 \mathrm{~mW}$, the lasing peak power rapidly increased and the single mode lasing was maintained with an enhanced SMSR of $\sim 20 \mathrm{~dB}$ represented as a solid line in figure 5. Beyond this power level, the quality factor (Q)s are gradually degraded in MMI coupled cavities due to a de-tuning effect. It is well known that the $\mathrm{Q}$ factors of the microcavities degrade as the pumping power exceeds the optimum power level. From the above result, we found that single mode operations can be maintained on the wide range of pump power.

\section{CONCLUSION}

Design and characteristics of the MMI coupled square cavities were demonstrated using InGaAsP MQW semiconductor layers. The MMI coupled square cavities exhibit single mode lasing spectra as expected. It was also found that the single resonance mode was preserved for a large ambient temperature range in spite of mode hopping. For the pump power change, the lasing spectrum was analyzed and the single mode lasing was maintained for the wide range of input optical power. From the experimental results, it was concluded that single mode lasing in MMI coupled square cavities can be obtained by relatively simple fabrication processes and the cavities can also be applied to any integrated passive or active building blocks in semiconductor devices.

\section{ACKNOWLEDGMENT}

This research was supported by the Basic Science Research Program through the National Research Foundation of Korea (NRF) funded by the Ministry of Education, Science and Technology (NRF-2010-0024686).

\section{REFERENCES}

1. R. Zhang, Z. Ren, and S. Yu, "Fabrication of InGaAsP double shallow ridge rectangular ring laser with total internal reflection mirror by cascade etching technique," IEEE Photon. Technol. Lett. 19, 1714-1716 (2007).

2. K.-H. Yoon, K. R. Oh, K. S. Kim, J. H. Kim, and K. C. Kim, "Monolithically integrated tunable laser using doublering resonators with a tilted multimode interference coupler," IEEE Photon. Technol. Lett. 21, 851-853 (2009).

3. A. Kapsalis, I. Stamataki, C. Mesaritakis, D. Syvridis, M. Hamacher, and H. Heidrich, "Design and experimental evaluation of active-passive integrated microring lasers: noise properties," IEEE J. Quantum Electron. 48, 99-106 (2012).

4. J. S. Parker, E. J. Norberg, Y.-J. Hung, B. Kim, R. S. Guzzon, and L. A. Coldren, "InP/InGaAsP flattened ring lasers with low-loss etched beam splitters," IEEE Photon. Technol. Lett. 23, 573-575 (2011).

5. G. Testa, Y. Huang, P. M. Sarro, L. Zemi, and R. Bernini, "Integrated silicon optofluidic ring resonator," Appl. Phys. Lett. 97, 131110 (2010).

6. S. Suzuki, Y. Kokubun, M. Nakazawa, T. Yamamoto, and S. T. Chu, "Ultrashort optical pulse transmission characteristics of vertically coupled microring resonator add/drop filter," J. Lightwave Technol. 19, 266-271 (2001).

7. H. Lee, G.-W. Kim, J.-O. Park, S.-H. Kim, and Y. Chung, "Widely tunable wavelength-selective reflector using polymer waveguide double-ring-resonator add/drop filter and loopback mirror," J. Opt. Soc. Korea 12, 157-161 (2008).

8. L. Shang, L. Liu, and L. Xu, "Single-frequency coupled asymmetric microcavity laser," Opt. Lett. 33, 1150-1152 (2008).

9. J. W. Ryu and M. Hentschel, "Ray model and ray-wave correspondence in coupled optical microdisks," Physical Review A 82, 033824 (2010).

10. D. G. Kim, G. Y. Oh, W. K. Choi, H. J. Kim, S. H. Kim, H. C. Ki, S. T. Kim, H. J. Ko, T. U. Kim, M. H. Yang, H. J. Kim, N. Dagli, and Y. W. Choi, "Compact triangular resonator without direct coupling between two access waveguides," Electron. Lett. 44, 1192-1193 (2008).

11. M. W. Kim and P.-C. Ku, "Semiconductor nanoring lasers," Appl. Phys. Lett. 98, 201105 (2011). 
12. S. C. Hagness, D. Rafizadeh, S. T. Ho, and A. Taflove, "FDTD microcavity simulations: design and experimental realization of waveguide-coupled single-mode ring and whispering-gallery-mode disk resonators," J. Lightwave Technol. 15, 2154-2165 (1997).

13. W. Green, R. Lee, G. DeRose, A. Scherer, and A. Yariv, "Hybrid InGaAsP-InP Mach-Zehnder racetrack resonator for thermooptic switching and coupling control," Opt. Express 13, 1651-1659 (2005).

14. T. J. Kippenberg, S. M. Spillane, and K. J. Vahala, "Demonstration of ultra-high-Q small mode volume toroid microcavitis on a chip," Appl. Phys. Lett. 85, 6113-6115 (2004).

15. A. Arbabi, Y. M. Kang, C.-Y. Lu, E. Chow, and L. L. Goddard, "Realization of a narrowband single wavelength microring mirror," Appl. Phys. Lett. 99, 091105-1 091105-3 (2011).

16. X. Zhang, H. Li, X. Tu, X. Wu, L. Liu, and L. Xu, "Suppression and hopping of whispering gallery modes in multiple-ring-coupled microcavity lasers," JOSA B 28, 483-488 (2011).

17. X. Zhang, L. Ren, X. Wu, H. Li, L. Liu, and L. Xu, "Coupled optofluidic ring laser for ultrahigh-sensitive sensing," Opt. Express 19, 22242-22247 (2011)

18. X. Wu, H. Li, L. Liu, and L. Xu, "Unidirectional singlefrequency lasing from a ring-spiral coupled microcavity laser,” Appl. Phys. Lett. 93, 081105 (2008).
19. J.-W. Ryu, S.-Y. Lee, and S. W. Kim, "Coupled nonidentical microdisks: avoided crossing of energy levels and unidirectional far-field emission," Phys. Rev. A 79, 053858 (2009).

20. H. Li, L. Shang, X. Tu, L. Liu, and L. Xu, "Coupling variation induced ultrasensitive label-free biosensing by using single mode coupled microcavity laser," J. Am. Chem. Soc. 131, 16612-16613 (2009).

21. H. Li and X. Fan, "Characterization of sensing capability of optofluidic ring resonator biosensors," Appl. Phys. Lett. 97, 011105 (2010).

22. K. Djordjev, S.-J. Choi, S.-J. Choi, and P. D. Dapkus, "High-Q vertically coupled InP microdisk resonators," IEEE Photon. Tech. Lett. 14, 331-333 (2002).

23. K.-S. Hyun and H.-J. Moon, "Resonant characteristics of multimode interferometer coupled square ring semiconductor resonators," Opt. Express 18, 6382-6389 (2010).

24. L. B. Soldano and E. C. M. Pennings, "Optical multi-mode interference devices based on self-imaging principles and applications," J. Lightwave Technol. 13, 615-627 (1995).

25. Y. Ma, S. Park, L. Wang, and S. T. Ho, "Ultracompact multimode interference 3-dB coupler with strong lateral confinement by deep dry etching," IEEE Photon. Technol. Lett. 12, $492-494$ (2000).

26. M. Kondow, T. Kitatami, K. Nakahara, and T. Tanaka, "Temperature dependence of lasing wavelength in a GaInNAs laser diode," IEEE Photon. Technol. Lett. 12, 777-779 (2000). 